\title{
Management of Demand Profiles on Mini-Grids in Developing Countries Using Timeslot Allocation
}

\author{
R. J. L. Gammon, P. J. Boait, V. Advani \\ De Montfort University \\ Leicester, United Kingdom \\ rgammon@dmu.ac.uk
}

\begin{abstract}
Stand-alone mini-grids provide vital energy access to rural communities across the Developing World where economic constraints necessitate optimal cost-effectiveness without compromising reliability or quality of service. Managing electricity demand to match supply availability - for example, by incentivizing consumers to operate loads at specific times can contribute to this aim. This paper addresses a method to achieve this, whereby timeslots are sold in which additional power is made available to participating consumers with highpowered, commercial loads, such as grain mills. Using a low-cost microprocessor to control remotely-switchable power sockets by wireless communications, circuits are activated according to the timeslots purchased without interruption of low-power (e.g. lighting and phone-charging) circuits. Informed by site survey data, laboratory tests demonstrated the system to be reliable and effective in maintaining demand closer to supply availability while avoiding overloads. This reduces losses and the need for storage while increasing energy access and return on investment.
\end{abstract}

Index Terms - Demand-side management, Developing World, Mini-grid, Timeslot allocation, Wireless remote switching.

\section{INTRODUCTION}

Currently, 1.2 billion people (about 17\% of the world's population) do not have access to electricity [1]. The vast majority of these are in rural communities in non-OECD countries. Electricity is so central to the functioning of modern lifestyles - or anything remotely approaching them - that economic wealth and quality of life are severely constrained by the lack of access to it [2]. This paper presents a technique that is aimed at reducing the cost of energy access and improving energy system reliability in rural mini-grids that provide electrical power to those not connected to national power networks.

A negative feedback loop persists in much of the Global South whereby the poor are unable to afford access to the electricity that could provide the very wealth-creating opportunities that would enable them to purchase electricity. One way to break this vicious cycle is to bring down the cost of the electricity supply such that more people can afford to access it. Hence, much foreign aid is focused on removing the burden of capital expenditure on energy systems in order to reduce the cost to electricity to users. However, this is not a sustainable situation as it can perpetuate a dependency culture in recipient communities and is contingent upon the continued generosity of donors. Rarely does it provide for the ongoing operational expenditure required in the management and maintenance of energy systems, leading to lack of accountability and inevitably to system failure [3]. One response has been to move towards a more fully commercial model that is economically self-sustaining [4], [5]. Economic return on investment (ROI) is critical to commercial success. Thus, the more revenue that can be derived from the operation of a mini-grid, the quicker the ROI. Better still, a more economically efficient system can offer a cheaper service to customers without slowing the rate of ROI.

In this paper we explore a demand-side management (DSM) technique that encourages consumers to shift their power demand to times that improve system reliability while reducing costs and maintaining the system operator's ROI. This is facilitated by offering customers with high power loads discounted rates for electricity consumption during timeslots allocated specifically to them. This work has been carried under the ESCoBox project, which is a collaboration between academics and practitioners in the field who are exploring the benefits of introducing simple 'smart grid' techniques to minigrids in the Developing World.

\section{BACKGROUND}

\section{A. Demand-Side Management}

Power supply and demand on any electricity network must be continuously maintained in perfect balance. On a conventional electricity network, typically powered by fossil fuels such as coal or gas, it has been relatively easy to manage this balance from the supply side, such that generation can follow the constantly changing energy demands of end-users by modulation of fuel input and power output. However, with the increasing use of renewable energy sources such as solar and wind, this supply-side management is more difficult achieve due to the stochastic nature of their outputs. This is driving a shift towards demand-side management (DSM), where demand is controlled in order to follow variations in variable renewable energy output. Scale has a smoothing effect, such that the variations of supply and demand aggregated across national-scale networks are proportionately less pronounced than those on mini-grids. Therefore the grid-
The authors would like to thank the Engineering and Physical Sciences Research Council (EPSRC), Department for International Development (DfID) and Department for Energy and Climate Change (DECC) for providing the financial support for this study under the ESCoBox (EP/L002566/1) project. 
balancing challenge is particularly acute in the mini-grids that are being deployed across the Developing World

Batteries are an obvious choice in mediating the supply and demand variabilities on mini-grids. However, batteries are not a simple panacea to this problem as they must be operated and maintained with great care in order to avoid rapid and costly degradation [6]. All too frequently, harsh operational regimes, especially those prevalent in the Developing World, lead to shortening of battery lifetimes, poor performance and ultimately in system failure. One answer is to install batteries of larger capacity to avoid the damaging depths of discharge that cause battery failure, but this is expensive and thus a particular challenge in poorer communities of the Global South. DSM, on the other hand, offers a means of avoiding deep discharge at considerably lower cost.

There are various techniques that fall under the category of DSM, including load shedding and time-shifting of demand. Judiciously deployed, these can mitigate the strain on batteries and thereby maintain their healthy state for longer, which can reduce the whole-life costs of the system as well as improve the reliability of energy delivery to users. Of the two, load shedding amounts to a limiting of access to electricity for certain users, albeit in an attempt to preserve that access to as many other users as possible. However, the time-shifting of demand loads does not prevent the consumption of the energy, but merely shifts it to a time that allows the electrical system as a whole to function more efficiently and reliably. Often this is achievable with little or no inconvenience, or discernable loss of utility, to the end user. Thus, time-shifting is the preferred option and therefore the subject of this paper.

The mini-grid systems being investigated under the ESCoBox project can be broadly divided into two categories: (1) Power-limited and (2) Energy-limited systems. Typically, hydro-electric schemes are power-limited as the limiting factor is the output capacity of the generator or the available water flow. The energy store (the watershed supplying the flow) is usually a less pressing constraint. Conversely, a solar or wind powered system is limited by the capacity of its battery (and the ability of the wind or solar resources to recharge that battery). In other words, blackouts occur on a power-limited network when the available generator output is overloaded and on an energy-limited network when the battery is at the limit of discharge. In the case of the former, time-shifting loads can prevent overload at peak demand periods and, in the latter, it can reduce reliance on the battery (with attendant losses) by scheduling demand to follow supply availability (i.e. during sunny or windy periods on solar or wind powered systems respectively). Another difference is that power-limited minigrids tend to be larger (10s-100s of $\mathrm{kW}$ ), whereas solar-based systems are rarely above $10 \mathrm{~kW}$ capacity (although this may change as cost fall further).

\section{B. Timeslot allocation}

Not all loads are amenable to shifting their time of operation, but in general, those that are have an intrinsic storage capability, be it of energy (as in a hot water tank) or material (as in a grain mill).

Lighting is considered a basic necessity that should be available whenever needed and so is not included in the timeslot allocation offer. Furthermore, the power consumption level of lighting, especially with LEDs, is too low to be of value in DSM. It would take a vast aggregation of lights (and the associated remote switching capabilities) to have any noticeable impact in demand shaping. Mobile phones have intrinsic storage capability and so would appear ideal for DSM; however, like lighting and other low power appliances, the power demand levels are too low for phone charging to have much impact in DSM. Therefore, all low-power devices are omitted from the timeslot allocation activity.

Moderately low-powered devices, such as refrigeration, entertainment (e.g. audio visual and music systems), hair dryers, water pumps, etc. tend to be used in commercial enterprises and these may have a contribution to make to DSM in some systems, particularly solar-based mini-grids, where larger loads are rare. Hefty loads, such as grain mills, welders, irrigation pumps, etc. are likely to be found on larger minigrids, for example, those powered by micro-hydro generators. These are good candidates for time-shifting.

Clearly, a mechanism is required that will persuade customers to participate in the proposed time-shifting of demand loads and this is usually incentivized by preferential tariffs. We are clear, however, that time-shifting should not be perceived as a limitation of service to customers, but rather as an option to buy additional service at low cost. Hence, we use the term 'timeslot allocation' to refer to timeslots for additional power consumption that are on offer to those wishing to purchase them. Thus, it is an additive mechanism, not a subtractive one. It is also important that the tariff structure and the practicalities of participation in the scheme are kept extremely simple. This is as true in the Developing World as much as in the Industrialized World [7].

\section{TECHNICAL IMPLEMENTATION}

There are two aspects to timeslot allocation in ESCoBox: the first is a control system that activates and deactivates circuits and the second is a Decision Support Tool (DST) that informs the allocation process. The complete system is illustrated in Fig 1.

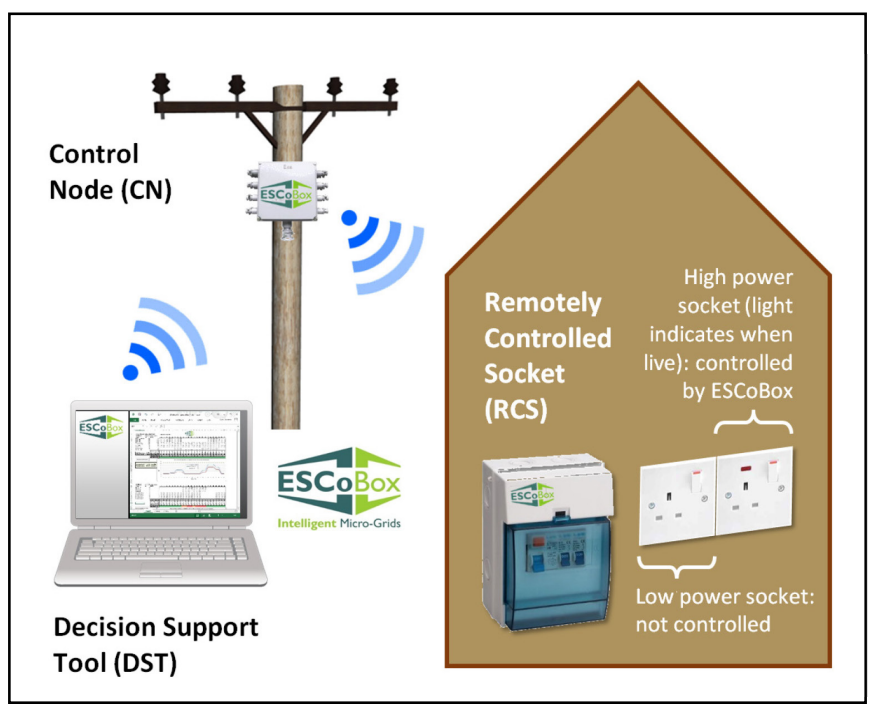

Figure 1. ESCoBox system overview 


\section{A. ESCoBox control system}

The ESCoBox system is designed to operate on its own or in conjunction with other mini-grid management systems, such as bitHarvester (developed by SteamaCo, who are a project partner), Conlog, etc.

At the heart of the ESCoBox system is a Control Node (CN) containing a Raspberry Pi microcontroller with a PiFace unit that uses Python scripts and a web-based graphic user interface to communicate wirelessly with up to six 13A remotely-controlled sockets (RCSs). For solar-based networks, 13A is sufficient to accommodate the larger loads found on such systems, which might be hair dryers, fridges, freezers, music and PA systems, etc. In communities with solar-based mini-girds, substantial loads like grain mills are likely to be driven by combustion engine, whereas they can be powered electrically on hydro-based schemes, which tend to have a higher generation capacity. In the latter case, the grain mill will draw considerably more than $13 \mathrm{~A}$ and so an RCS can be used to activate relay switches of suitable size to carry the power necessary for it, which might be around $10-25 \mathrm{~kW}$ and running on 3 phases. On networks with more than 6 highpowered appliances participating in the timeslot-allocation scheme, additional CNs, each with a Raspberry Pi controller, can be daisy-chained together with power-line Ethernet used to communicate with each other.

In some cases, the remote switching element of ESCoBox may be omitted. For instance, SteamaCo manages their systems largely through SMS messages sent to the bitHarvester unit or to customers' mobile phones, for example, warning them that their credit is running low. Switching off customers is only a last resort, e.g. for non-payment. In such a system, the timeslot allocation might not be enforced by remote switching, but by advisory SMS messages that inform customers if they draw power outside their allocated timeslot and warning them of the additional cost it would incur. This approach is more suited to an energy-limited system, whereas the mandatory control of timeslots by remote switching is appropriate on a power-limited system to prevent system overload and resulting blackouts.

\section{B. ESCoBox Decision Support Tool}

ESCoBox's Decision Support Tool (DST) is designed to assist the designer or operator of a mini-grid system in estimating the demand profile of the system and the effect that the time-shifting of loads has on it, including the revenue obtained. Thus, it can contribute to the technical efficiency and resilience of the system and de-risk investment decisions in the implementation or ongoing growth of the system.

To maximize accessibility, the tool has been developed in Microsoft Excel with a VBA (Visual Basic for Applications) macro. It is also designed to be used in conjunction with the widely used micro-grid design software HOMER. The core calculation that the ESCoBox DST uses is a Monte Carlo simulation of the electricity demand generated by a given population of appliances and their probability of being in operation in each hour of the day [8]. The ESCoBox DST is published on the ESCoBox project website [9]. The usual caveat for free software applies, which is that it is offered in the hope that it is useful but no assurance of fitness for purpose is given.

Initially, a baseline demand curve is set up, whereby the number of each type of appliance is combined with the probability of that appliance being switched on at each hour of the day, as in the example shown in Table I. This produces the graph shown in Fig 2, plus a value for daily revenue, which is dependent upon the tariff set by the mini-grid system operator (MSO). The MSO can also set an approximate risk factor, related to the probability of overload occurring ( 1 in 1,000, 1 in 10,000 , etc.), and can set the run length of the Monte Carlo simulation $(100,1,000$, or 10,000$)$. The resultant graph shows the average and peak demand profiles (red and blue lines respectively). The dashed green line represents the maximum power available, which is a straight line in this case as it is a hydro-electric generator. In a solar or wind powered system, this would follow anticipated supply profiles informed by empirical data.

Table II shows an example of how the MSO might wish to sell allocated timeslots for high power demand loads. These are entered into the Controlled Appliance table in the same way as had been done in the Uncontrolled Appliance table.

TABLE I. BASELINE DEMAND PROFILE CONFIGURATION TABLE

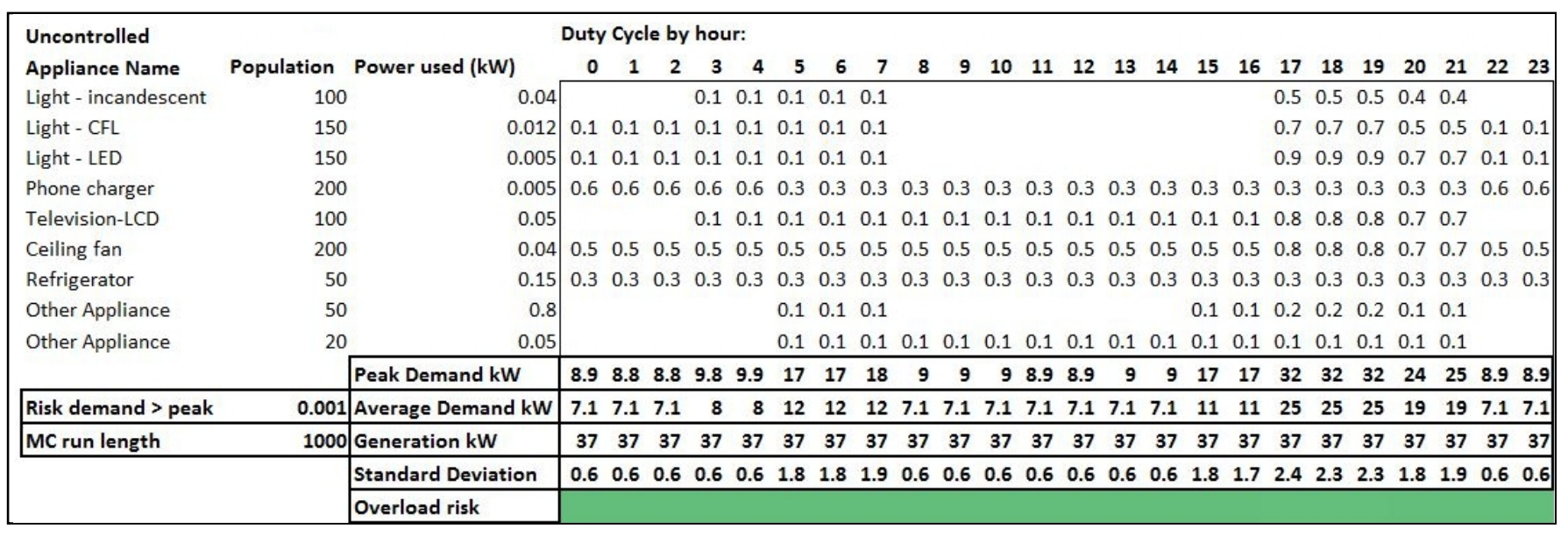




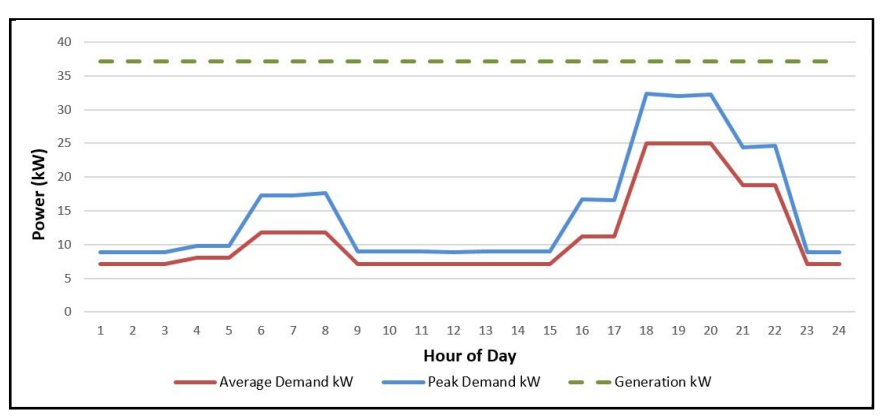

Figure 2. Baseline peak and average demand daily profile

Another graph is produced, as illustrated in Fig 3, which shows the anticipated effect of adding these loads in their allotted timeslots. Again, a revenue value is calculated, which takes account of a different tariff level that can be set for the controlled loads. The bottom row of both tables displays a red, orange, or green color block for each timeslot to highlight the level of risk of overload. In the example shown in Table II and Fig 3, the risk of overload has been deliberately set to a level that illustrates this. Fig 4 shows how shortening the timeslot offer (00:00-07:00 and 20:00-24:00) to irrigation pumps and slightly staggering the two maize mills (Mill 1 gets 10:0016:00 and Mill 2 gets 08:00-14:00) reduces the risk of overload significantly.

As illustrated in Fig 4, once the MSO is satisfied with the demand profile created by the sale of timeslots, the information can be uploaded wirelessly from the DST to the
$\mathrm{CN}(\mathrm{s})$ for execution.

The DST can be employed at the design stage of mini-grid implementation to provide a model of consumer demand as an input to system sizing. Later, operational data and experience can be used to fine-tune the assumptions made in the initial model parameterized by the Uncontrolled and Controlled Appliance tables. As part of the ESCoBox project, our partners at Newcastle University are developing load disaggregation and battery management techniques to enhance this aspect of the system. Latent demand is generally high across the Developing World [10] so, once established, a mini-grid is likely to continue to expand. As an MSO prepares to take on more customers and generator capacity, the ESCoBox DST offers a valuable tool in guiding such growth.

\section{RESULTS AND IMPACT}

Laboratory tests show the consistent reliability of wireless switching operations at ranges up to $30 \mathrm{~m}$ from a Raspberry $\mathrm{Pi}$ control node. Therefore, control nodes will need to be positioned such that they can reach a cluster of up to 6 businesses that participate in the timeslot allocation scheme. The DST fulfils the requirements set out by ESCoBox project partners in that it offers:

- A mechanism for planning DSM activities that can improve the technical reliability and efficiency of minigrids as well as their economic performance. Comparing Figs 2 and 4, it is clear that the addition of several high-power loads can be achieved with minimal

TABLE II. TIMESLOT ALLOCATION TABLE

\begin{tabular}{|c|c|c|c|c|c|c|c|c|c|c|c|c|c|c|c|c|c|c|c|c|c|c|c|c|c|c|}
\hline \multirow{2}{*}{$\begin{array}{l}\text { Controlled } \\
\text { Appliance Name }\end{array}$} & \multirow{2}{*}{$\begin{array}{l}\text { ESCoBox } \\
\text { switch no. }\end{array}$} & \multirow[b]{2}{*}{ Power used (kW) } & \multicolumn{5}{|c|}{ Duty Cycle by hour: } & \multirow[b]{2}{*}{5} & \multirow[b]{2}{*}{6} & \multirow[b]{2}{*}{7} & \multirow[b]{2}{*}{$\varepsilon$} & \multirow[b]{2}{*}{9} & \multirow[b]{2}{*}{10} & \multirow[b]{2}{*}{11} & \multirow[b]{2}{*}{12} & \multirow[b]{2}{*}{13} & \multirow[b]{2}{*}{14} & \multirow[b]{2}{*}{15} & \multirow[b]{2}{*}{16} & \multirow[b]{2}{*}{17} & \multirow[b]{2}{*}{18} & \multirow[b]{2}{*}{19} & \multirow[b]{2}{*}{20} & \multirow[b]{2}{*}{21} & \multirow[b]{2}{*}{22} & \multirow[b]{2}{*}{23} \\
\hline & & & 0 & 1 & 2 & 3 & 4 & & & & & & & & & & & & & & & & & & & \\
\hline Hair dryer 1 & & 1 & & & & & & & & & 0.1 & 0.1 & 0.1 & 0.1 & 0.1 & 0.1 & 0.1 & 0.1 & 0.1 & & & & & & & \\
\hline Hair dryer 2 & & 1 & & & & & & & & & 0.1 & 0.1 & 0.1 & 0.1 & 0.1 & 0.1 & 0.1 & 0.1 & 0.1 & & & & & & & \\
\hline Maize mill 1 & & 12 & & & & & & & & & 0.2 & 0.2 & 0.2 & 0.2 & 0.2 & 0.2 & 0.2 & 0.2 & 0.2 & & & & & & & \\
\hline Maize mill 2 & & 12 & & & & & & & & & 0.2 & 0.2 & 0.2 & 0.2 & 0.2 & 0.2 & 0.2 & 0.2 & 0.2 & & & & & & & \\
\hline Irrigation pump 1 & & 2 & 0.5 & 0.5 & 0.5 & 0.5 & 0.5 & 0.5 & 0.5 & 0.5 & 0.5 & 0.5 & 0.5 & 0.5 & 0.5 & 0.5 & 0.5 & & & & & & 0.5 & 0.5 & 0.5 & 0.5 \\
\hline Irrigation pump 2 & & 2 & 0.5 & 0.5 & 0.5 & 0.5 & 0.5 & 0.5 & 0.5 & 0.5 & 0.5 & 0.5 & 0.5 & 0.5 & 0.5 & 0.5 & 0.5 & & & & & & 0.5 & 0.5 & 0.5 & 0.5 \\
\hline Irrigation pump 3 & & 1 & 0.5 & 0.5 & 0.5 & 0.5 & 0.5 & 0.5 & 0.5 & 0.5 & 0.5 & 0.5 & 0.5 & 0.5 & 0.5 & 0.5 & 0.5 & & & & & & 0.5 & 0.5 & 0.5 & 0.5 \\
\hline Irrigation pump 4 & & 1 & 0.5 & 0.5 & 0.5 & 0.5 & 0.5 & 0.5 & 0.5 & 0.5 & 0.5 & 0.5 & 0.5 & 0.5 & 0.5 & 0.5 & 0.5 & & & & & & 0.5 & 0.5 & 0.5 & 0.5 \\
\hline \multirow[t]{6}{*}{ Irrigation pump 5} & & 1 & 0.5 & 0.5 & 0.5 & 0.5 & 0.5 & 0.5 & 0.5 & 0.5 & 0.5 & 0.5 & 0.5 & 0.5 & 0.5 & 0.5 & 0.5 & & & & & & 0.5 & 0.5 & 0.5 & 0.5 \\
\hline & & Peak Demand kW & 16 & 16 & 16 & 17 & 17 & 23 & 23 & 23 & 37 & 37 & 38 & 37 & 38 & 37 & 37 & 38 & 39 & 32 & 32 & 32 & 30 & 30 & 16 & 16 \\
\hline & & Average Demand kW & 11 & 11 & 11 & 12 & 12 & 15 & 15 & 15 & 16 & 16 & 16 & 16 & 16 & 16 & 16 & 16 & 16 & 25 & 25 & 25 & 22 & 22 & 11 & 11 \\
\hline & & Generation kW & 37 & 37 & 37 & 37 & 37 & 37 & 37 & 37 & 37 & 37 & 37 & 37 & 37 & 37 & 37 & 37 & 37 & 37 & 37 & 37 & 37 & 37 & 37 & 37 \\
\hline & & Standard deviation & 1.7 & 1.7 & 1.8 & 1.8 & 1.7 & 2.5 & 2.4 & 2.5 & 7 & 7 & 7.2 & 6.9 & 7.3 & 6.9 & 7.1 & 7.2 & 7.2 & 2.3 & 2.3 & 2.4 & 2.4 & 2.4 & 1.7 & 1.7 \\
\hline & & Overload risk & & & & & & & & & & & & & & & & & & & & & & & & \\
\hline
\end{tabular}

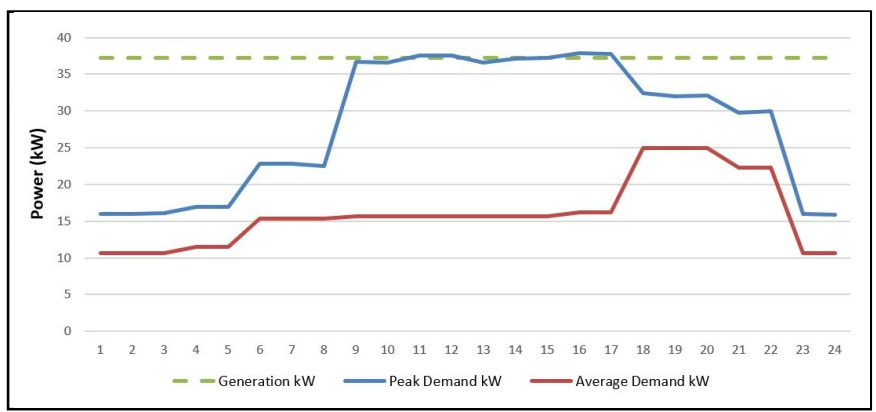

Figure 3. Peak and average demand daily profile with timeslot allocated loads

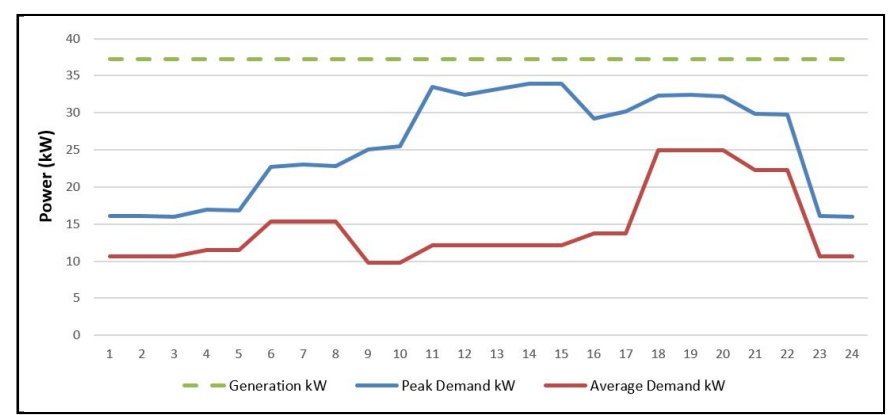

Figure 4. Peak and average demand daily profile with timeslot allocation adjusted to reduce risk of overload 
risk of overload, allowing the generator to operate at higher capacity factor to increase ROI and reduce costs to customers.

- A tool for assisting in the design and expansion of mini-grids

- A means of predicting with greater certainty the potential success of offering tariff incentives to promote DSM

- A means of reducing the risk to investors in the implementation of mini-grids

The combination of the ESCoBox DST and the control system enables an MSO to plan and implement DSM measures that improve the technical and economic performance of a mini-gird system. The hardware costs are modest, with a single control node, consisting a Raspberry Pi, Piface, 6 wirelessly controlled sockets, enclosures and other minor accessories. Even to a small business, this is a minor investment that will quickly recoup the cost. The cost to the MSO of having access to the DST is that of a basic PC or laptop, plus the Microsoft Excel software. This is a modest investment in comparison with the cost of installing the energy system itself, yet its effect on the performance and viability of that system may be very significant.

Clearly, this system relies on a degree of business acumen and human initiative on the part of the MSO. It is a decision 'support' tool, not a decision 'making' tool. As in any business, success is highly dependent upon understanding the customers. In this case, the MSO must apply that knowledge (about local conditions, business practice, culture, specific customer needs, etc.) to the entering of appliance populations and duty cycle data into the tables and take a view on what level of risk of system overload is acceptable.

\section{CONCLUSION}

The ESCoBox Decision Support Tool (DST) and control system has been developed in order to improve the technical and economic performance of mini-grids so that access to electricity is increased in off-grid communities across the Developing World. By lowering the cost of building and operating mini-grids, while simultaneously making them more resilient, the ESCoBox system lowers the barriers to electricity access in some of the world's poorest communities. The system allows a Mini-grid System Operator (MSO) to incentivize flexibility of demand that improves their return on investment (ROI) by enabling the system to run closer to full capacity, for longer periods, while diminishing the risk of overload and blackouts. This is achieved by offering high power users preferential rates to draw power at certain times of the day, week, or year. The DST helps the MSO to decide what timeslots can be offered to which users and at what price in order to optimize the system's techno-economic performance and reliability. The worked example provided in this paper illustrates how the ESCoBox system works and confirms its functionality in the laboratory. Once the proposed test site has recovered from the combination of drought and flood damage that has delayed a field trial, this will be verified in the real world.

\section{ACKNOWLEDGMENT}

The authors wish to thank all ESCoBox project partners: Newcastle University, the University of Nairobi, the Institute of Development Studies, SteamaCo, Practical Action Consulting, BBOXX and Ashden. We gratefully acknowledge the contributions of many community members in Kenya, Malawi, Nepal, The Gambia, Zimbabwe and Rwanda who have contributed vital knowledge and first-hand experience to the ESCoBox project team.

\section{REFERENCES}

[1] International Energy Agency, "World Energy Outlook 2015 Electricity database". Available: http://www.worldenergyoutlook.org/resources/en ergydevelopment/energyaccessdatabase/

[2] B. K. Sovacool, "The political economy of energy poverty: A review of key challenges", Energy for Sustainable Development, vol. 16, pp. 272282, Jun. 2012.

[3] J. E. Hazelton, A.G. Bruce, I. F. MacGill, "A review of the potential benefits and risks of photovoltaic hybrid mini-grid systems", Renewable Energy, vol. 67, pp. 222-229, Jul. 2014.

[4] P. R. Krithika and D. Palit, "Participatory Business Models for Off-grid Electrification", in Rural Electrification Through Decentralised Offgrid Systems in Developing Countries, S. Bhattacharyya Ed. London: Springer-Verlag, 2013, Chapter 8.

[5] Alliance for Rural Electrification, "Hybrid Mini-grids for rural electrification: lessons learned". Available: http://www.ruralelec.org/ 38.0.html\#c1936, 2011

[6] P. Ruetschi, "Aging mechanisms and service life of lead-acid batteries", Journal of Power Sources, vol. 127, pp. 33-44 Mar. 2004.

[7] A. Faruqui and S.Sergici, "Household response to dynamic pricing of electricity: a survey of 15 experiments", $J$ Regul Econ., vol. 38, pp. 193-225, Oct. 2010.

[8] P. Boait, V. Advani, R. Gammon, "Estimation of demand diversity and daily demand profile for off-grid electrification in developing countries", Energy for Sustainable Development, vol. 29, pp. 135-141, Dec. 2015.

[9] Boait P. ESCoBox ESCoBox Decision Support Tool, 2016. Available: http://www.dmu.ac.uk/research/research-faculties-and-institutes/institut e-of-energy-and-sustainable-development/research-projects/escobox. aspx.

[10] C. Wolfram, O. Shelef, P. J. Gertler, "How Will Energy Demand Develop in the Developing World?", J Econ Perspectives, vol. 26, pp. 119-138, Jan. 2012. 